\title{
Clinical Trial Period
}

National Cancer Institute

\section{Source}

National Cancer Institute. Clinical Trial Period. NCI Thesaurus. Code C71737.

A possibly-repeating collection of consecutive days that define the length of a Study Segment 